\title{
Synthesis of CdS nanostructure by Lawesson's reagent for photocatalytic water splitting
}

\author{
Vikram U. Pandit*, Sudhir S. Arbuj, Uttam P. Mulik, Suresh W. Gosavi and Bharat B. Kale \\ Centre for Materials for Electronics Technology (C-MET), Off Pashan Road, \\ Panchwati, Pune-411008, Maharashtra, India \\ Email:*vikramupandit@gmail.com,\#kbbb1@yahoo.com
}

Recently, nanostructured materials have been at the centre of attention owing to their fascinating properties. All these properties include optical, electronic, thermal, mechanical, chemical, and physical properties. These nanomaterials display unique properties not only from their bulk counterparts but also from those of the atomic or molecular precursors from which they are synthesized. In this context, cadmium sulfide with a band gap $2.4 \mathrm{eV}$ is a promising candidate owing to its wide ranging applications in nonlinear optical devices, flat-panel displays, light-emitting diodes, lasers, logic gates, and transistors.

The CdS in bulk as well as nano form makes it an attractive semiconductor for photocatalytic applications like water splitting.

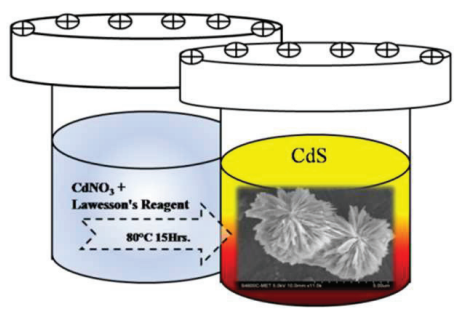

Figure 1: Synthesis of CdS nanostructure

Hydrogen is an ideal fuel for future owing to its clean and renewable nature. Hydrogen can be produced by gasification from biomass, methane reforming, through the electrolysis of $\mathrm{H}_{2} \mathrm{O}$ photocatalytic cleavage of $\mathrm{H}_{2} \mathrm{O}$. Among all the available catalysts, $\mathrm{CdS}$ is a promising photocatalyst for $\mathrm{H}_{2} \mathrm{O}$ splitting due to suitable position of the conduction band edge that is more negative than the reduction potential of $\mathrm{H}^{+} / \mathrm{H}_{2}$.

Herein, CdS nanostructures produced by using an inexpensive and simple procedure (shown in Figure 1) with Lawesson's reagent as a sulfur source for visible-light driven photocatalytic hydrogen generation from $\mathrm{H}_{2} \mathrm{O}$. The formation of carnation flower like morphology mechanism has shown in Figure 2. The photocatalytic hydrogen generation was carried out using prepared $\mathrm{CdS}$ nanostructures in presence of three different sacrificial reagents (Figure 3) viz., a) methanol b) $0.1 \mathrm{M} \mathrm{Na}_{2} \mathrm{~S}$ and 0.04 $\mathrm{M} \mathrm{Na} \mathrm{SO}_{3}$ and c) $2 \mathrm{ml}$ Benzyl alcohol dissolved in $5 \mathrm{ml}$ acetic acid.

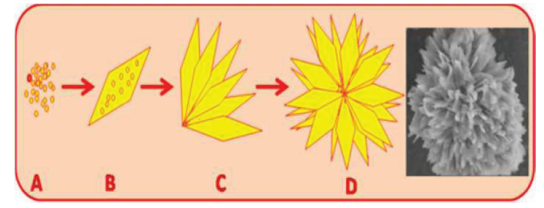

Figure 2: Formation Mechanism of $\mathrm{CdS}$ nanostructure

The stability of the photocatalyst is already confirmed by performing recycle experiments. The chemistry of photo corrosion is well understood in case of CdS for water splitting. However, in case of $\mathrm{H}_{2} \mathrm{~S}$ splitting reaction in presence of sacrificial reagents, the photo-corrosion process is hindered and ultimately the formation of $\mathrm{Cd}$ ions also negligible.

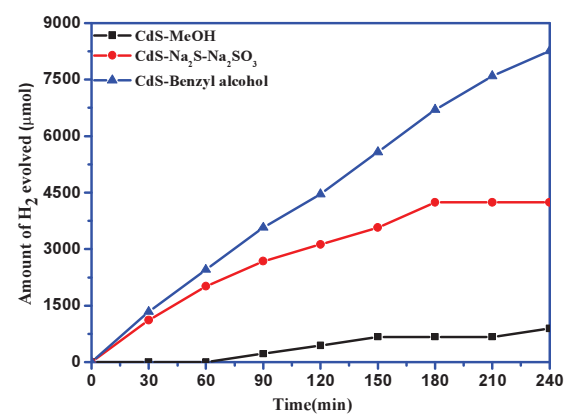

Figure 3: Photocatalytic $\mathrm{H}_{2}$ generation via $\mathrm{H}_{2} \mathrm{O}$ splitting

Hence, under the given experimental conditions, the presence of a sacrificial agent, an electron sink in the form of $\mathrm{Pt}$ on the surface and the size of the photocatalyst particles have perceptible influence on the photocatalytic hydrogen production.

\section{References}

1. F. E. Osterloh, Chem. Soc. Rev. 42, (2013), 2294. 2. S. R. Lingampalli, U.K. Gautam, C. N. R. Rao, Energy Environ. Sci. 6, (2013), 3589.

3. J. K. Vaishnav, S. S. Arbuj, S. B. Rane, D. P. Amalnerkar, RSC Adv., 4, (2014), 47637.

4. V. U. Pandit, S. S. Arbuj, R. R. Hawaldar, P. V. Kshirsagar, J. D. Ambekar, U. P. Mulik, S. W. Gosavi, B. B. Kale RSC Adv., 5, (2015), 13715. 